\title{
Multivariable robust control of a proton exchange membrane fuel cell system
}

\author{
Fu-Cheng Wang*, Hsuan-Tsung Chen, Yee-Pien Yang, Jia-Yush Yen \\ Department of Mechanical Engineering, National Taiwan University, Taipei 10617, Taiwan \\ Received 10 September 2007; received in revised form 15 November 2007; accepted 15 November 2007 \\ Available online 23 November 2007
}

\begin{abstract}
This paper applies multivariable robust control strategies to a proton exchange membrane fuel cell (PEMFC) system. From the system point of view, a PEMFC can be modeled as a two-input-two-output system, where the inputs are air and hydrogen flow rates and the outputs are cell voltage and current. By fixing the output resistance, we aimed to control the cell voltage output by regulating the air and hydrogen flow rates. Due to the nonlinear characteristics of this system, multivariable robust controllers were designed to provide robust performance and to reduce the hydrogen consumption of this system. The study was carried out in three parts. Firstly, the PEMFC system was modeled as multivariable transfer function matrices using identification techniques, with the un-modeled dynamics treated as system uncertainties and disturbances. Secondly, robust control algorithms were utilized to design multivariable $H_{\infty}$ controllers to deal with system uncertainty and performance requirements. Finally, the designed robust controllers were implemented to control the air and hydrogen flow rates. From the experimental results, multivariable robust control is shown to provide steady output responses and significantly reduce hydrogen consumption.
\end{abstract}

(C) 2007 Elsevier B.V. All rights reserved.

Keywords: Proton exchange membrane fuel cell; Multivariable control; Robust control; System identification

\section{Introduction}

In recent years, alternative energy resources have gained more and more attention due to the greenhouse effect and the decreasing levels of fossil fuel. Among them, the proton exchange membrane fuel cell (PEMFC) is an important candidate for replacing traditional fuel because of its favorable characteristics, including low operation temperature, fast power response, high power density, low noise pollution, high system efficiency and environmental friendliness. Until recently, PEMFC has been applied to many systems such as vehicles, boats, etc. [1,2]. For most of the applications, batteries and dc/dc converters were utilized to provide steady output voltages. However, the use of those peripheral components can decrease the efficiency of the fuel cell system. On the other hand, traditional control methodologies such as process control were frequently applied to PEMFC.

\footnotetext{
* Corresponding author. Tel.: +8862 33662680; fax: +886223631755.

E-mail addresses: fcw@ntu.edu.tw (F.-C. Wang),d89522001@ntu.edu.tw (H.-T. Chen), ypyang@ntu.edu.tw (Y.-P. Yang), jyen@ccms.ntu.edu.tw (J.-Y. Yen).
}

Nevertheless, these control methods cannot provide good system performance and may cause instability due to the nonlinear characteristics of the fuel cell system. Therefore, in this paper we consider the closed-loop structures of the PEMFC system, and apply robust control strategies to improve system stability and performance.

Forrai et al. [3] applied system identification methods to model a PEMFC system as a circuit consisting of inner resistors and a capacitor. Kazim and Lund [4] performed a basic parametric study of a PEMFC system, and showed that the system performance can be improved at lower cell temperature and higher cell pressure with a higher air stoichiometric ratio. Wang et al. [5] discussed a distributed generation system and designed a controller to maintain the power delivered from the fuel cell system to the utility grid. The simulation results showed that the designed proportional-integral (PI) controller could maintain system stability even with some system faults. Woo and Benziger [6] designed a proportional-integral-derivative (PID) controller to regulate the hydrogen flow rate and tuned the oxygen flow at a ratio of 1.3:2 $\left(\mathrm{O}_{2}: \mathrm{H}_{2}\right)$ to obtain optimal performance. VegaLeal et al. [7] developed a multi-input-single-output (MISO) system to control the output current. They designed a feed- 
forward controller to adjust the airflow rate, and a proportional controller to regulate temperature so that the net power is optimized. Methekar et al. [8] considered a multi-input-multi-output (MIMO) system with inputs of hydrogen and coolant and outputs of power density and temperature, and proposed two PID control strategies. The simulation results showed that the ratio control strategy achieved a faster response than the MIMO control strategy. Rodatz et al. [9] illustrated a dynamic model of air supply for a PEMFC system. They designed a linear-quadraticGaussian (LQG) controller to decouple the pressure trace from the mass flow trace, which provided better performance than PI control. Di Domenico et al. [10] extended this idea to create a multi-variable LQG controller, designed to tune the excess air ratio while tracking the optimal pressurization to maximize system efficiency for transient loads. Sedghisigarchi and Feliachi [11] designed an $H_{\infty}$ controller to regulate the cell voltage under small load variations. From the simulations, the output voltage offset was kept below $5 \%$ by controlling the hydrogen flow rate.

Many studies have utilized hybrid systems to improve overall system performance. In those systems, fuel cell was regarded as the main power source, which was combined with other auxiliary power sources to provide steady output power. Thounthong et al. [12] integrated a fuel cell and super-capacitors for electric vehicles. They aimed to control the transient power through PID control of the super-capacitors, while the fuel cell operated at a steady rate. Lee et al. [13] designed a hybrid system for a vehicle equipped with a fuel cell and a battery. The system power was supplied by the fuel cell at low loadings and by the battery at high loadings when the output voltage of fuel cell stack was too low. Jurado and Saenz [14] developed an adaptive controller for a fuel-cell micro-turbine hybrid power plant. Taking into account the system variation and disturbances, they found that the controller, designed at a fixed operating point, could stabilize the system under different operating conditions. For some applications, the dc/dc converters were designed to increase system efficiency. Wai et al. [15] employed the voltage-clamped and soft-switching techniques to design a dc/dc converter. The experimental results illustrated that the converter can achieve more than $95 \%$ efficiency for a $250 \mathrm{~W}$ PEMFC system. Zenith and Skogestad [16] utilized sliding mode control to adjust the duty cycle of a rapid dc/dc converter to control the output voltage. Jiang et al. [17] developed a system consisting of a fuel cell, a battery and a dc/dc converter. By adjusting the duty cycle of the converter, they found that the battery could be charged by the fuel cell through either the maximum power strategy or the maximum efficiency strategy.

Because a steady power source is important for electrical equipment, in this paper robust control methodologies are utilized to guarantee a steady voltage or current supply when the operating conditions change. Robust control is well known for its capability in dealing with system uncertainties and disturbances [18-20]. Wang et al. [21-23] applied robust control to a single-input-single-output (SISO) PEMFC system to achieve steady voltage output by regulating the oxygen flow rate. The experimental results illustrated that robust controllers can cope with system perturbations and achieve splendid performance. Furthermore, the robust control can also replace the dc/dc con- verter and broaden the applications. This paper extends $H_{\infty}$ control strategies to a multi-input fuel-cell system, where the proposed multivariable robust controllers can provide steady voltage and reduce hydrogen consumption by regulating the air and the hydrogen flow rates simultaneously. The experimental results show that the designed MISO robust controllers are better than the SISO controller in terms of hydrogen consumption and energy dissipation. This paper is arranged as follows: in Section 2, fuel-cell dynamics is described and modeled as a MIMO system. The Pulse Width Modulation (PWM) theory is also introduced and applied to control the hydrogen valve. In Section 3, we utilize robust control strategies to design multivariable $H_{\infty}$ controllers. In Section 4 , the designed controllers are implemented to verify their performance. Finally, we draw some conclusions in Section 5.

\section{Materials and methods}

In this section, fuel cell dynamics is described and modeled as a MIMO system using identification techniques. Those system matrices will then be used for the controller designs in Section 3.

\subsection{System description}

The fuel-cell system considered in this paper was designed and manufactured by Chung Shan Institute of Science and Technology (CSIST) and integrated by DELTA Electronics ${ }^{\text {TM }}$. The inputs of the system are hydrogen and air while the outputs are cell voltage and current. The system consists of 15 cells with an active area of $50 \mathrm{~cm}^{2}$ on each. The maximum efficiency of the fuel cell stack is $37 \%$ (Lower Heating Value, LHV) under dry $\mathrm{H}_{2}$ /air and humidification-free conditions [22].

The dynamics of the fuel-cell system is non-linear and timevarying in that it is influenced by many factors, including the diffusion dynamic, the Nernst equation, proton concentration dynamics and cathode kinetics as follows:

diffusion equation : $\quad R_{\mathrm{ohm}}=R_{\mathrm{ref}}+\alpha_{T}\left(T-T_{\mathrm{ref}}\right)$,

Nernst equation : $\quad E=E_{\mathrm{ref}}+\frac{\mathrm{d} E}{\mathrm{~d} T}\left(T-T_{\mathrm{ref}}\right)$

$$
+k \frac{\mathrm{RT}}{2 F} \ln \left(P_{\mathrm{H}_{2}} P_{\mathrm{O}_{2}}^{1 / 2}\right),
$$

proton concentration dynamics :

$u\left(\frac{-\partial C_{\mathrm{H}^{+}}}{\partial t}\right) \frac{\partial C_{\mathrm{H}^{+}}}{\partial t}+\frac{C_{\mathrm{H}^{+}}}{\tau_{\mathrm{H}^{+}}}=\frac{1+\alpha_{\mathrm{H}^{+}} j^{3}}{\tau_{\mathrm{H}^{+}}}$,

cathodic kinetics : $\quad \eta=b \ln \left\{\frac{p_{10}\left[\mathrm{H}^{+}\right]_{0}}{p_{1}\left[\mathrm{H}^{+}\right]}\left(1+\frac{j_{r}}{j_{0} A_{r}}\right)\right\}$,

as shown in Fig. 1 [24].

From the system point of view, the physics-based model of Fig. 1 can be represented as a MIMO system, as depicted in 


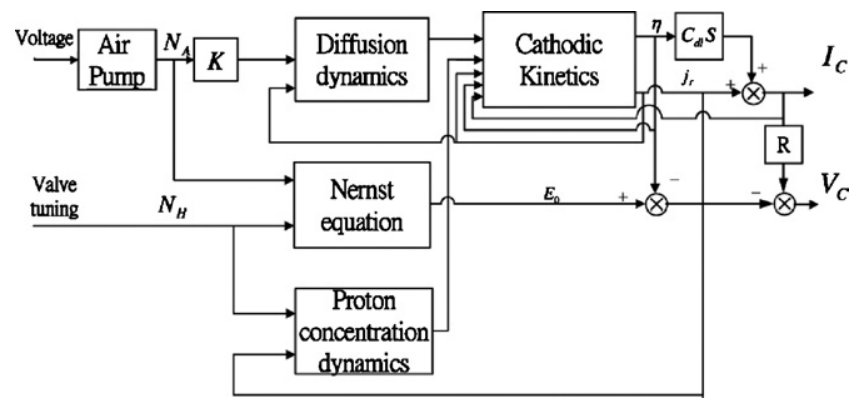

Fig. 1. Dynamics of the PEMFC system [24].

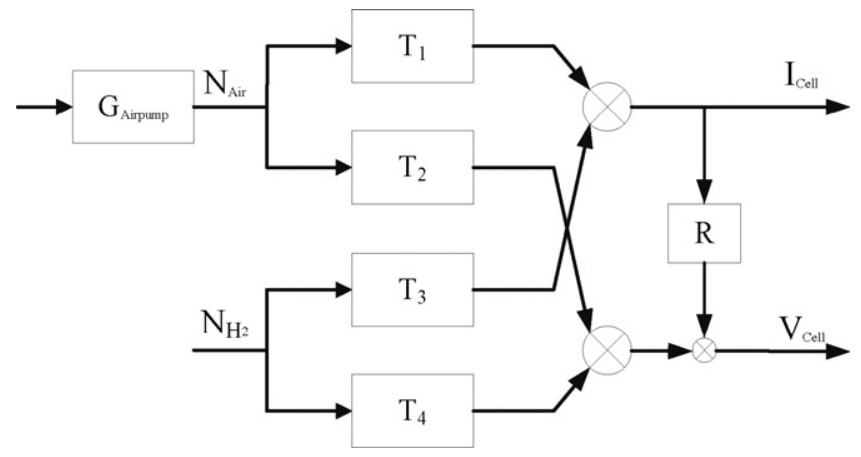

Fig. 2. The block diagram of the fuel cell system [22].

Fig. 2, with the following relation [25]:

$I_{\text {cell }}=T_{1}(s) N_{\text {air }}+T_{3}(s) N_{\mathrm{H}_{2}}$,

$V_{\text {cell }}=T_{2}(s) N_{\text {air }}+T_{4}(s) N_{\mathrm{H}_{2}}-R I_{\text {cell }}$,

in which $T_{1}(s) \sim T_{4}(s)$ represent the transfer functions of the system. It is noted that the dynamics of the linearized model of (5) and (6) depends on the operating conditions. For example, when the current load varies from 2 to $6 \mathrm{~A}$, the output voltage decreases significantly from 11 to $7.5 \mathrm{~V}$ using the on-board controller, as illustrated in Fig. 3. Therefore, robust control algorithms are applied to achieve steady outputs even when the operating conditions change. By fixing the output resistance, we can either control the cell voltage or current output by regulating the air $\left(N_{\text {air }}\right)$ and the hydrogen $\left(N_{\mathrm{H}_{2}}\right)$ flow rates. Since most electrical equipment requires constant voltage supply, in this paper we aim to control the cell voltage output.

\subsection{System identification}

In order to describe the transfer functions of (5) and (6), we measured the input and output signals of the fuel-cell system,

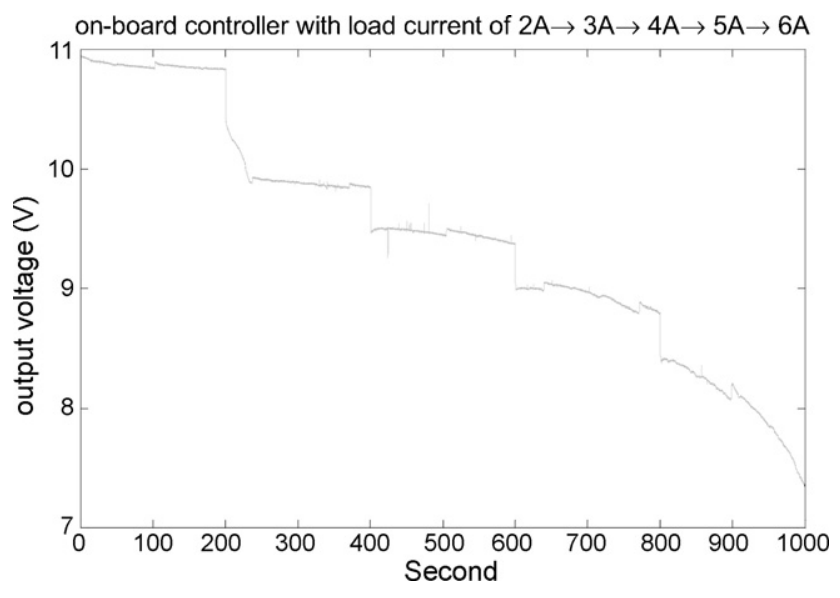

Fig. 3. Voltage variations when the current loading is changed.

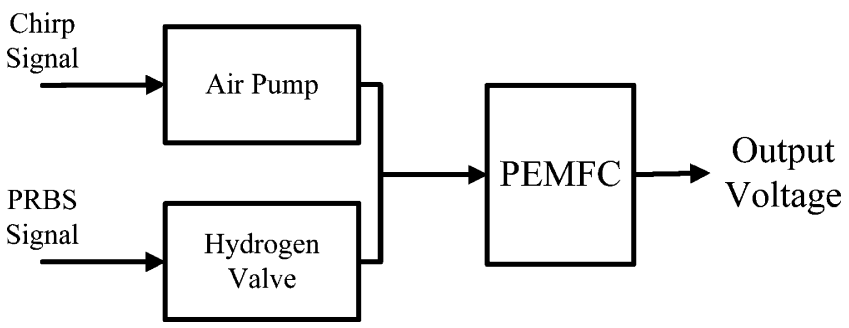

Fig. 4. The MISO PEMFC system for system identifications.

and utilized subspace system identification methods to estimate the models in state-space form, as presented in the following:

$\left\{\begin{array}{l}x_{t+1}=A x_{t}+B u_{t}+v_{t} \\ y_{t}=C x_{t}+D u_{t}+v_{t}\end{array}\right.$,

in which $u_{t} \in \mathbb{R}^{m}$ and $y_{t} \in \mathbb{R}^{l}$ are the input and output signals, while $x_{t} \in \mathbb{R}^{n}$ represents the state and $v_{t} \in \mathbb{R}^{n}, v_{t} \in \mathbb{R}^{l}$ are zero mean white Gaussian noise vector sequences. By applying the numerical algorithms for subspace state space system identification (N4SID), the linear model of (7) can be derived by the low-rank approximation of a matrix obtained from a set of component-wise least squares support vector machines regression problems [26].

For the experiments, a chirp signal and a pseudorandom, binary signal (PRBS) were generated to control the air pump and the hydrogen valve of the PEMFC system, respectively, as shown in Fig. 4. Both the frequencies of the chirp signal and PRBS were set at $0.01-5 \mathrm{~Hz}$ (see Fig. 5(a)). We set the current loadings as $2 \mathrm{~A}, 3 \mathrm{~A}$ and $4 \mathrm{~A}$, and measured the output voltage responses, as illustrated in Fig. 5(b). In order to

Table 1

Transfer functions at the operation points

\begin{tabular}{|c|c|c|c|c|c|c|}
\hline & $2 \mathrm{~A}$ & & $3 \mathrm{~A}$ & & $4 \mathrm{~A}$ & \\
\hline 1 & $G_{11}=\left[\frac{0.00202 z-0.001598}{z^{2}-1.954 z+0.9555}\right.$ & $\left.\frac{0.000505 z-0.0003996}{z^{2}-1.954 z+0.9555}\right]$ & $G_{21}=\left[\frac{0.001935 z-0.00153}{z^{2}-1.971 z+0.973}\right.$ & $\left.\frac{0.0004837 z-0.0003824}{z^{2}-1.971 z+0.973}\right]$ & $G_{31}=\left[\frac{0.001603 z-0.001052}{z^{2}-1.934 z+0.9373}\right.$ & $\left.\frac{0.0004 z-0.0002629}{z^{2}-1.934 z+0.9373}\right]$ \\
\hline 2 & $G_{12}=\left[\frac{0.00156 z-0.001158}{z^{2}-1.976 z+0.9771}\right.$ & $\left.\frac{0.0003901 z-0.0002896}{z^{2}-1.976 z+0.9771}\right]$ & $G_{22}=\left[\frac{0.001919 z-0.001483}{z^{2}-1.974 z+0.9753}\right.$ & $\left.\frac{0.0004798 z-0.0003708}{z^{2}-1.974 z+0.9753}\right]$ & $G_{32}=\left[\frac{0.001774 z-0.001231}{z^{2}-1.932 z+0.9354}\right.$ & $\left.\frac{0.0004435 z-0.0003077}{z^{2}-1.932 z+0.9354}\right]$ \\
\hline
\end{tabular}


(a)
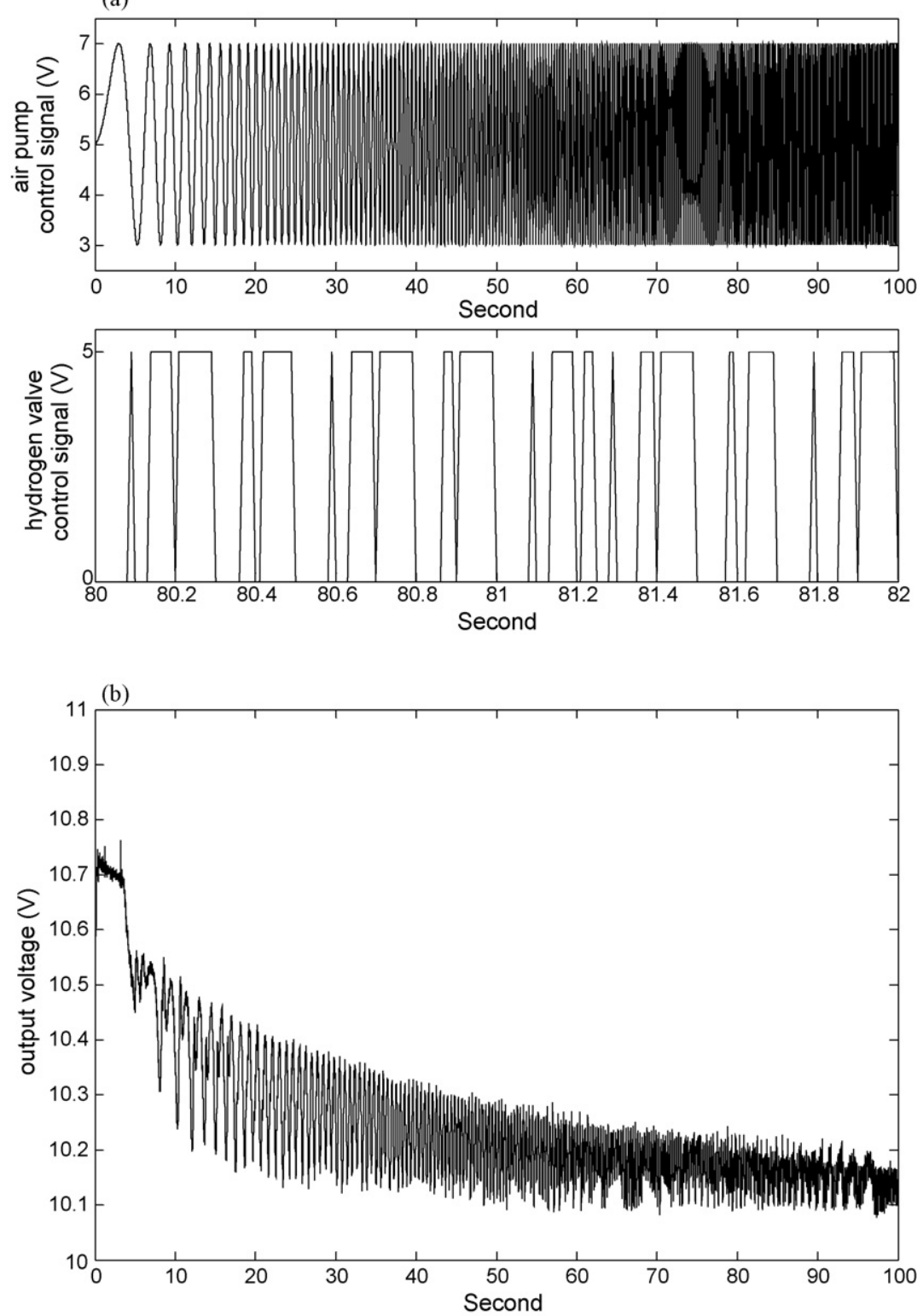

Fig. 5. The input and output signals of the system (with $3 \mathrm{~A}$ load): (a) the input signals and (b) the output voltage responses.

take system variation into account, we repeated the experiments three times at each operating condition, and employed the aforementioned identification techniques to obtain the corresponding transfer functions illustrated in Table 1. Those transfer functions will be utilized for robust controller design in Section 3.

\subsection{Pulse Width Modulation theory}

To control the hydrogen valve, the PWM theory is employed. In recent years, the combination of PWM and fast-switch valves has been widely applied in many control fields, such as position control of pneumatic actuators [27]. PWM is a modulation tech- (a)

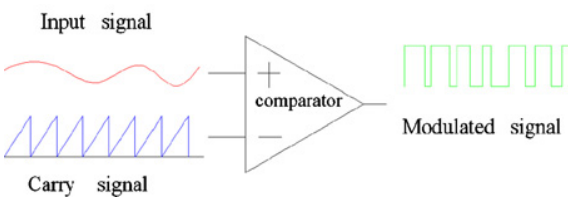

(b)

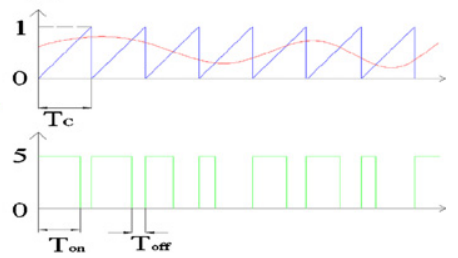

Fig. 6. Working principle of Pulse Width Modulation: (a) illustrations and (b) the signals. 


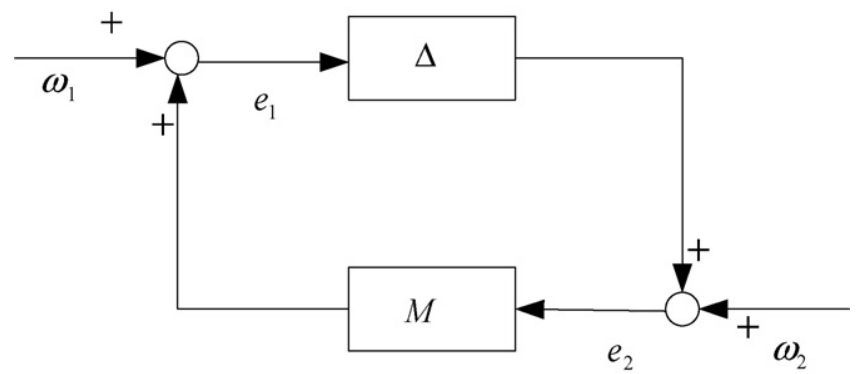

Fig. 7. Illustration of Small Gain Theorem.

nique which utilizes a carry function to generate variable-width pulses in order to represent the amplitude of an input signal. As illustrated in Fig. 6, given the input signal and carry function, a comparator is utilized to compare the magnitudes of these two signals to generate the modulated signal. When the input signal is greater than the carry signal, the modulated signal is set to be "high". Otherwise, it is set to be "low". In Fig. 6(b), the period of the modulated signal is the same as the period of the carry signal, $T_{c}$. Furthermore, the duty ratio $\tau$ of the modulated signal is defined as

$\tau=\frac{T_{\mathrm{on}}}{T_{c}}$,

in which $T_{\text {on }}$ is the operating time. In applying PWM to control the hydrogen valve of the fuel-cell system, more hydrogen is supplied when $\tau$ is increased. In general, the frequency and amplitude of the carry signal must be higher than those of the input signals. To control hydrogen flow, we employed a $2 / 2$-way MAC 35A-AAA-DAA-1BA valve with a switch frequency of about $1 \mathrm{kHz}$ and a maximum power consumption of $5.4 \mathrm{~W}$ [28]. For the experiments, we utilized a chirp signal from $0.01-5 \mathrm{~Hz}$, and a carry function with a frequency of $10 \mathrm{~Hz}$ and maximum amplitude of $1 \mathrm{~V}$. Using the comparator, a PRBS signal, such as Fig. 5(a), was generated to control the hydrogen valve.

\section{Theory and calculation}

In this section, the robust control algorithms are introduced, and applied to design $H_{\infty}$ controllers to provide the maximum stability bound for the fuel cell system. The resulting controllers are then implemented with PWM to verify the effect by experimentation in Section 4.

Theorem 1 (Small Gain Theorem [20]). Suppose $M \in R H_{\infty}$ and let $\gamma>0$, then the interconnected system shown in Fig. 7, where $\omega_{i}$ represent the input signals and $e_{i}$ the error signals, is well posed and internally stable for all $\Delta(s) \in R H_{\infty}$ with (a) $\|\Delta\|_{\infty} \leq 1 / \gamma$ if and only if $\|M(s)\|_{\infty} \leq \gamma ;(b)\|\Delta\|_{\infty}<1 / \gamma$ if and only if $\|M(s)\|_{\infty}<\gamma$, where $\|G\|_{\infty}$ is the $\infty$ norm of system $G$.

Suppose that a nominal plant $G_{0}$ can be expressed as $G_{0}=M^{-1} N$, where (1) $M, N \in R H_{\infty}$ and (2) $M M^{*}+N N^{*}=I$, $\forall \omega$. This is called a normalised left coprime factorisation of $G_{0}$. Furthermore, suppose that a perturbed system $G_{\Delta}$ can be

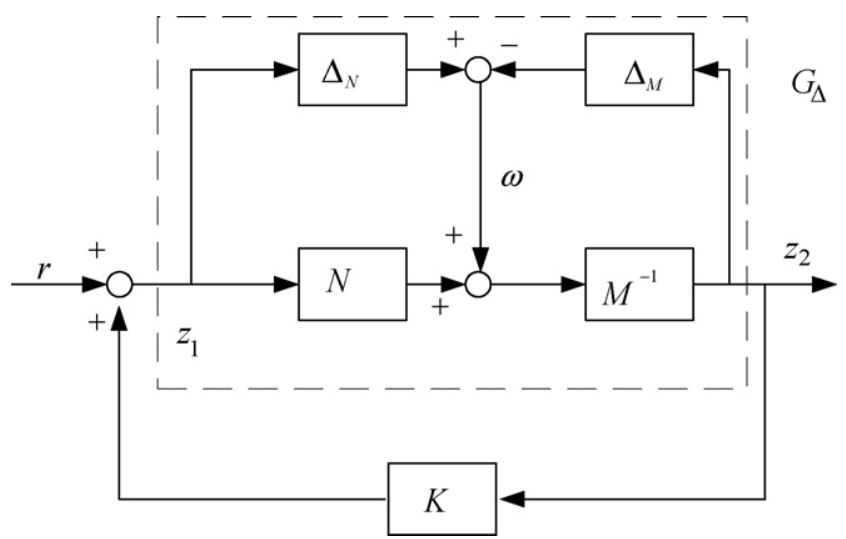

Fig. 8. Feedback structure of the perturbed plant $G_{\Delta}$ with a controller $K$.

expressed as

$G_{\Delta}=\left(M+\Delta_{M}\right)^{-1}\left(N+\Delta_{N}\right)$,

with $\left\|\left[\Delta_{M}, \Delta_{N}\right]\right\|_{\infty}<\varepsilon, \Delta_{M}, \Delta_{N} \in R H_{\infty}$. Considering a controller $K$ with the block structure of Fig. 8 where $z_{i}$ and $\omega$ are corresponding input and output signals of the systems, the system transfer functions can be simplified as follows:

$$
\begin{aligned}
{\left[\begin{array}{l}
z_{1} \\
z_{2}
\end{array}\right] } & =\left[\begin{array}{c}
K \\
I
\end{array}\right](I-G K)^{-1} M^{-1} \omega \\
& =\left[\begin{array}{c}
K \\
I
\end{array}\right](I-G K)^{-1}\left[\begin{array}{ll}
I & G
\end{array}\right] \omega,
\end{aligned}
$$

as shown in Fig. 9. Therefore, from Theorem 1, the closed-loop system remains internally stable for all $\left\|\left[\Delta_{M}, \Delta_{N}\right]\right\|_{\infty}<\varepsilon$ if and only if

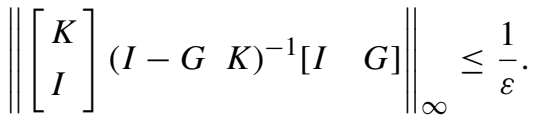

Thus we can define the stability margin of the system in following:

Definition 1 (Stability Margin [29]). The stability margin $b(G$, $K$ ) of the closed-loop system is defined as follows:

$b(G, K) \equiv\left\|\left[\begin{array}{l}K \\ I\end{array}\right](I-G K)^{-1}\left[\begin{array}{ll}I & G\end{array}\right]\right\|_{\infty}^{-1}$.

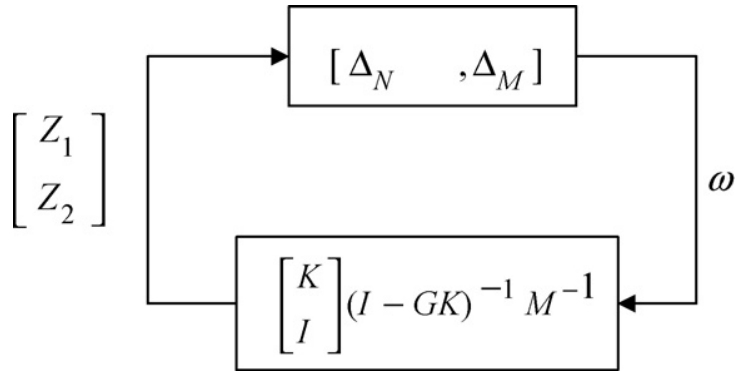

Fig. 9. Reconfiguration of the closed-loop system. 
Hence, from Theorem 1 the closed-loop system is internally stable for all $\left\|\left[\Delta_{M}, \Delta_{N}\right]\right\|_{\infty}<\varepsilon$ if and only if $b(G, K) \geq \varepsilon$.

It is further noted that the coprime factorisation of a system is not unique. That is, there is more than one expression for $G_{0}$ or $G_{\Delta}$. Therefore, the gap between two systems $G_{0}$ and $G_{\Delta}$ can be defined as

Definition 2 (Gap Metric [20]). The smallest value of $\left\|\left[\Delta_{M}, \Delta_{N}\right]\right\|_{\infty}$ which perturbs $G_{0}$ into $G_{\Delta}$, is called the gap between $G_{0}$ and $G_{\Delta}$, and is denoted as $\delta\left(G_{0}, G_{\Delta}\right)$.

\subsection{Selection of the nominal plant}

From the definitions, $b(G, K)$ gives the radius (in terms of gap metric) of the largest ball of plants stabilized by the controller $K$. Therefore, the goal of the controller design is to derive a suitable controller $K$ from a nominal plant $G_{0}$, such that all perturbed plants $G_{i}$ located inside the gap $\delta\left(G_{0}, G_{i}\right)<\varepsilon$ will satisfy $b(G$, $K) \geq \varepsilon$ and the closed-loop system will remain internally stable.

The selection of the nominal plants $G_{0}(s)$ was based on the calculation of gaps between the nominal plants and the perturbed plants, such that the maximum gap is minimized as

$\min _{G_{0}} \max _{G_{i}} \delta\left(G_{0}, G_{i}\right)$

Considering the system transfer function matrices in Table 1, the gaps between all plants are illustrated in Table 2. Therefore, $G_{23}$ was selected as the nominal plant because the maximum gap between $G_{23}$ and other plants is 0.2449 , which is the minimum of all systems. The maximum gap can be regarded as the maximum perturbation of the system due to the changes of operating conditions, such as temperature, humidification and power loads.

\subsection{Robust controller synthesis}

The design procedures of the robust controller are illustrated as follows [30]:

(1) Loop shaping design: as shown in Fig. 10(a), the nominal plant $G$ is shaped by a pre-compensator $W_{1}$ and a post-

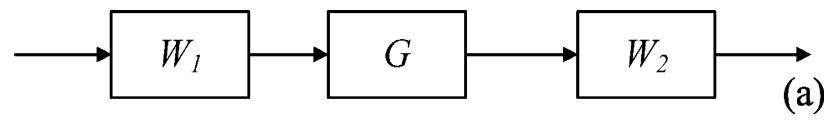

(a)
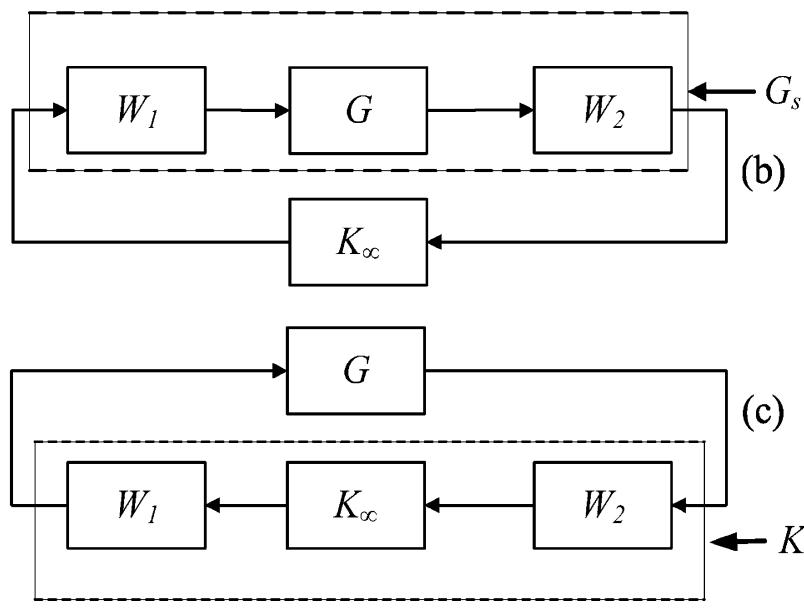

Fig. 10. The design procedures of robust controllers.

compensator $W_{2}$ to form a shaped plant $G_{S}=W_{2} G W_{1}$.

(2) Robust stabilization estimate: the maximum stability margin $b_{\max }$ is defined as follows:

$b_{\max }\left(G_{S}, K\right) \triangleq \inf _{K}\left\|\left[\begin{array}{l}K \\ I\end{array}\right]\left(I-G_{S} K\right)^{-1}\left[\begin{array}{ll}I & G_{S}\end{array}\right]\right\|_{\infty}^{-1}$,

where $M_{s}, N_{s}$ are the normalized left coprime factorization of $G_{s}$, i.e. such that $G_{s}=M_{s}^{-1} N_{s}$. If $b_{\max }\left(G_{s}\right.$, $K) \ll 1$, then we must return to step (1) and modify $W_{1}$ and $W_{2}$. Finally, we can select an $\varepsilon \leq b_{\max }\left(G_{s}, K\right)$ and synthesize a stabilizing controller $K_{\infty}$, which satisfies $\left\|\left[\begin{array}{l}K_{\infty} \\ I\end{array}\right]\left(I-G_{s} K_{\infty}\right)^{-1}\left[\begin{array}{ll}I & G_{S}\end{array}\right]\right\|_{\infty}^{-1} \geq \varepsilon$, as shown in Fig. 10 (b).

(3) The designed controller $K_{\infty}$ is then multiplied by the weight functions, such that $K=W_{1} K_{\infty} W_{2}$ is implemented to control the system $G$, as illustrated in Fig. 10(c).

Table 2

Gaps of the plants

\begin{tabular}{|c|c|c|c|c|c|c|c|c|c|}
\hline & $G_{11}$ & $G_{12}$ & $G_{13}$ & $G_{21}$ & $G_{22}$ & $G_{23}$ & $G_{31}$ & $G_{32}$ & $G_{33}$ \\
\hline$G_{11}$ & 0 & 0.2127 & 0.1346 & 0.1278 & 0.3054 & 0.0751 & 0.078 & 0.0966 & 0.0956 \\
\hline$G_{12}$ & 0.2127 & 0 & 0.3395 & 0.2098 & 0.2137 & 0.1649 & 0.2858 & 0.3034 & 0.3044 \\
\hline$G_{13}$ & 0.1346 & 0.3395 & 0 & 0.2068 & 0.4254 & 0.1932 & 0.0585 & 0.039 & 0.0488 \\
\hline$G_{21}$ & 0.1278 & 0.2098 & 0.2068 & 0 & 0.3522 & 0.1327 & 0.161 & 0.1785 & 0.1922 \\
\hline$G_{22}$ & 0.3054 & 0.2137 & 0.4254 & 0.3522 & 0 & 0.2449 & 0.3736 & 0.3902 & 0.3844 \\
\hline$G_{23}$ & 0.0751 & 0.1649 & 0.1932 & 0.1327 & 0.2449 & 0 & 0.1366 & 0.1551 & 0.1522 \\
\hline$G_{31}$ & 0.078 & 0.2858 & 0.0585 & 0.161 & 0.3736 & 0.1366 & 0 & 0.0195 & 0.0341 \\
\hline$G_{32}$ & 0.0966 & 0.3034 & 0.039 & 0.1785 & 0.3902 & 0.1551 & 0.0195 & 0 & 0.0263 \\
\hline$G_{33}$ & 0.0956 & 0.3044 & 0.0488 & 0.1922 & 0.3844 & 0.1522 & 0.0341 & 0.0263 & 0 \\
\hline Max & 0.3054 & 0.3395 & 0.4254 & 0.3522 & 0.4254 & 0.2449 & 0.3736 & 0.3902 & 0.3844 \\
\hline
\end{tabular}

Bold values signify the selection of the nominal plant, which is the minimization of the maximum system gaps, as illustrated in Eq. (13). 


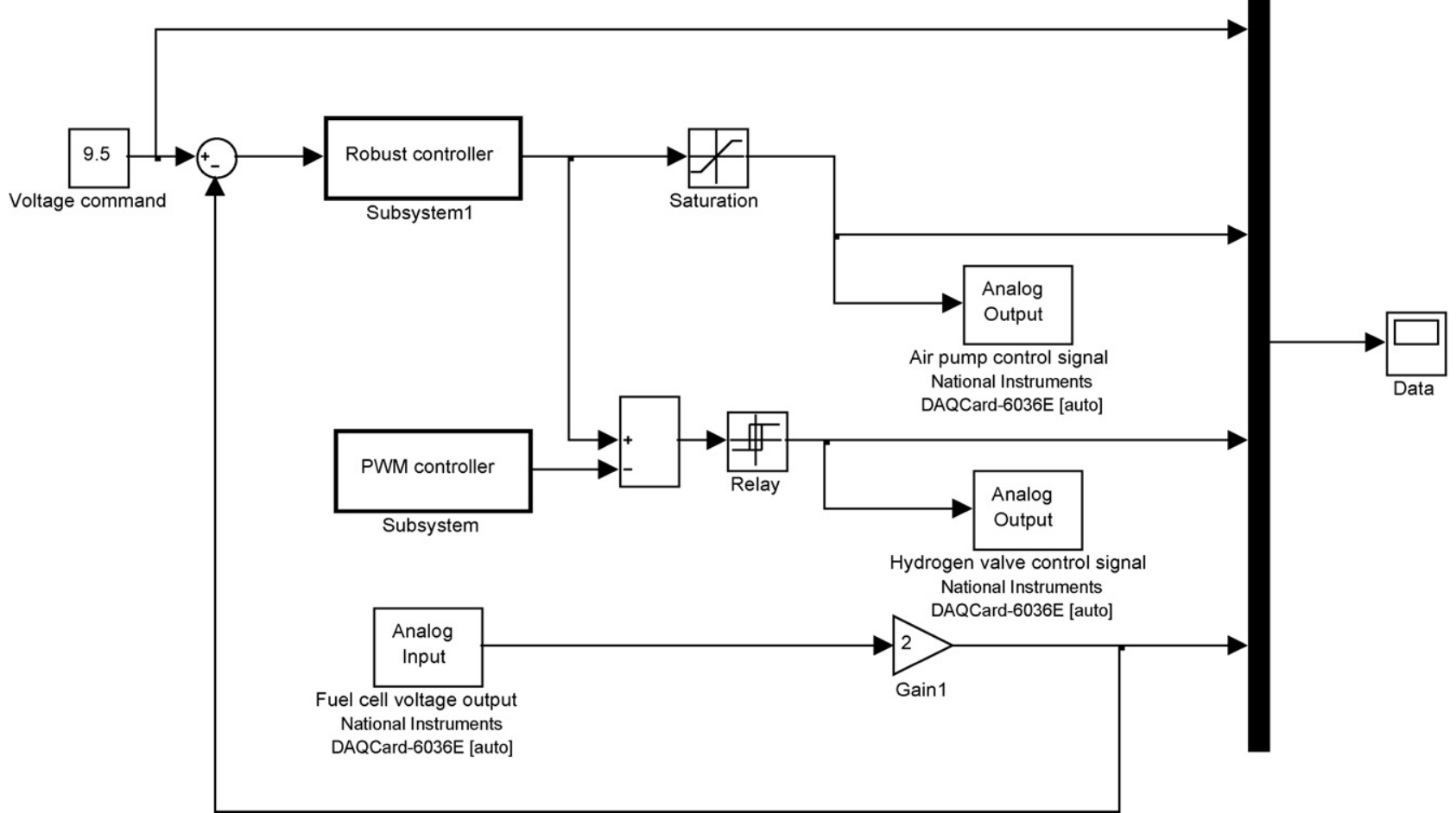

Fig. 11. The control structure in Matlab/Simulink.

For the first design, we employed $G_{23}(z)$ :

$G_{23}(z)=\left[\begin{array}{ll}\frac{0.00154 z-0.000985}{z^{2}-1.948 z+0.95} & \frac{0.0003851 z-0.0002462}{z^{2}-1.948 z+0.95}\end{array}\right]$,
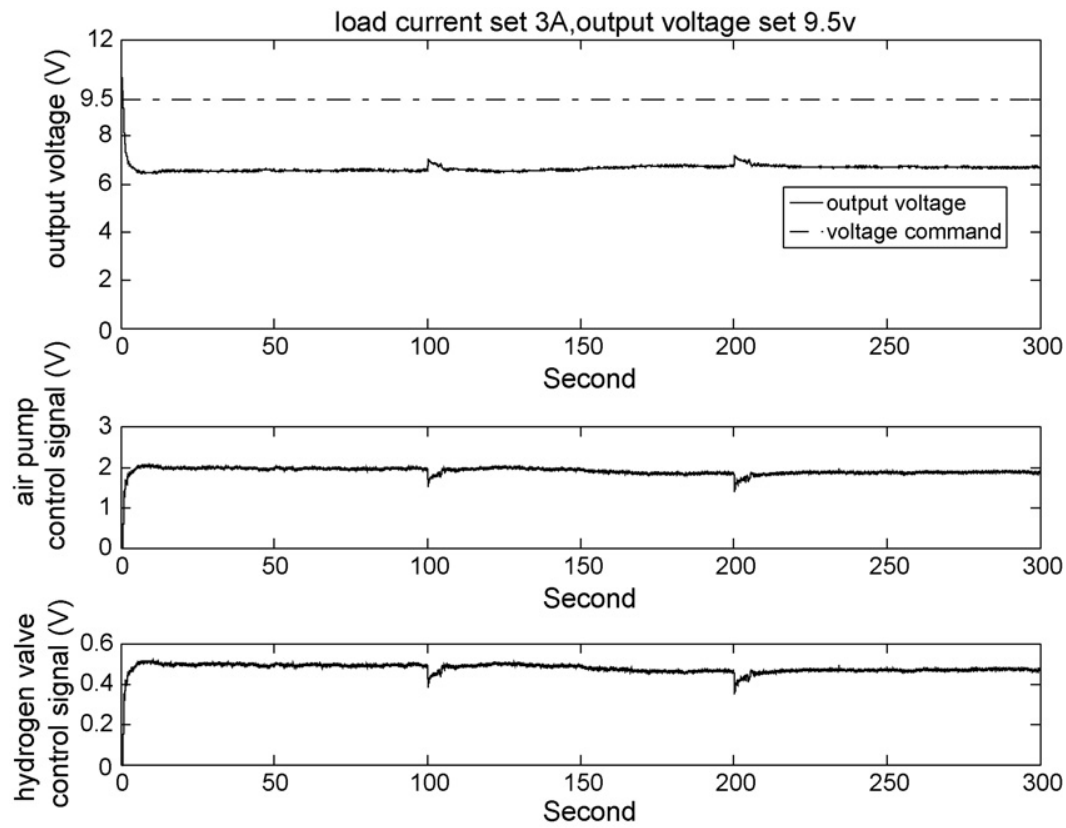

Fig. 12. The voltage responses and control signals with $K_{23}(z)$. 
The optimal (in terms of the stability bound) $H_{\infty}$ controller $K_{23}(z)$ was designed as

$K_{23}(z)=\left[\begin{array}{c}\frac{-0.5769 z+0.5586}{z-0.9183} \\ \frac{-0.1442 z+0.1397}{z-0.9183}\end{array}\right]$,

which gives a stability bound of $b\left(G_{23} W_{1}, K_{23}\right)=0.8595$. The stability bound is much larger than the maximal gap (0.2449) of the systems. Therefore, the controller can easily stabilize the system even with plant perturbations. Implemented with $W_{1} K_{23}(z)$, the experimental results showed a significant steady-state error in the voltage output. This is because the current design emphasized on stability rather than performance. Therefore, to improve system performance, we need to add integrals in the weighting functions to eliminate the steady-state error [31,chapter 9].

For the second design, the following weighting function

$W_{1}^{\prime}(z)=\left[\begin{array}{ll}\frac{z-0.99}{z-1} & 0 \\ 0 & \frac{0.006}{z-1}\end{array}\right]$,

was utilized to eliminate the steady-state errors of the output responses. Following the procedures, a $H_{\infty}$ robust controller

(a)
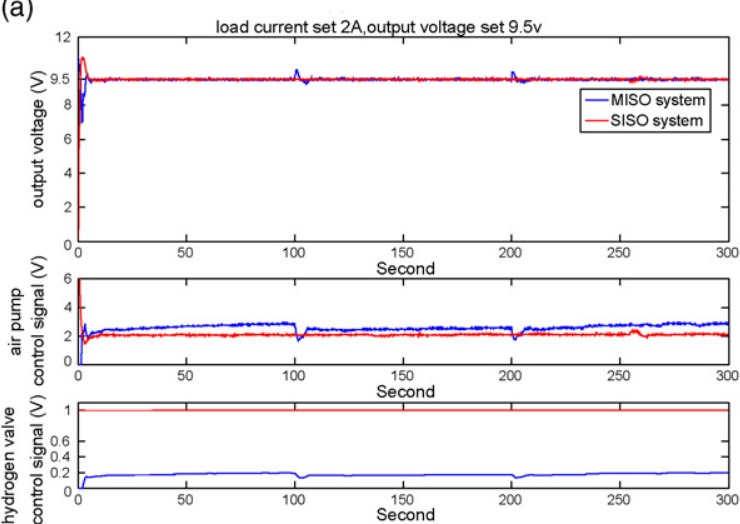

(c)
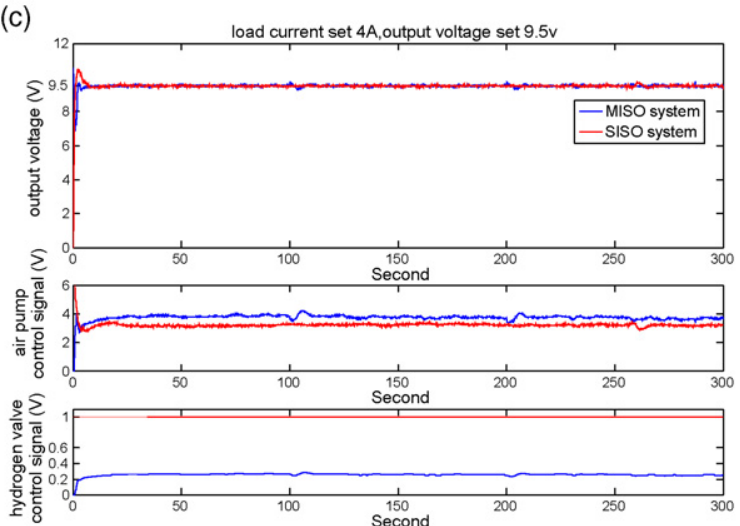

was designed as

$K_{23}^{\prime}(z)=\left[\begin{array}{c}\frac{-0.8446 z^{2}+1.647 z-0.804}{z^{2}-1.941 z+0.9422} \\ \frac{-0.08869 z^{2}+0.1728 z-0.08427}{z^{2}-1.941 z+0.9422}\end{array}\right]$,

with a stability bound of $b\left(G_{23} W_{1}^{\prime}, K_{23}^{\prime}\right)=0.7622$, which is still greater than the maximum gap $(0.2449)$ of the systems, but less than the previous design (0.8595). However, the integral in $W_{1}^{\prime}(z)$ guaranteed a zero steady-state error in the output responses. Therefore, the choice of weighting functions can be regarded as a compromise between system performance and stability, such that the designed controller $K_{23}^{\prime}(z)$ can achieve robust performance for the fuel cell system.

\section{Results and discussion}

In order to implement the controllers, Matlab ${ }^{\mathrm{TM}}$ was employed with a data-acquisition (DAQ) card to control the PEMFC system. The control structure in Matlab/Simulink is illustrated in Fig. 11.

Implemented with $W_{1} K_{23}(z)$ and the voltage command set at $9.5 \mathrm{~V}$, the output voltage responses are shown in Fig. 12. Firstly, the voltage output was about $6.6 \mathrm{~V}$ with some perturbation when the water was purged. Secondly, it is as expected that the system achieved excellent stability but poor performance, with a root-mean-square (RMS) error of about $2.8 \mathrm{~V}$. Furthermore, we
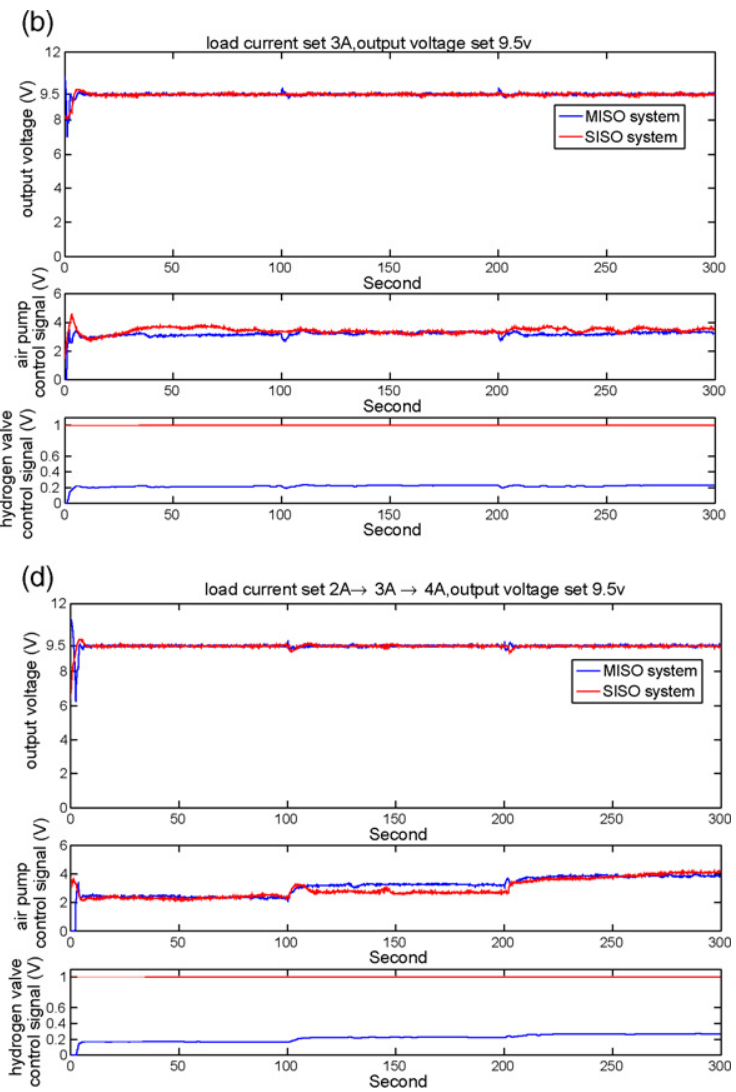

Fig. 13. The comparison of voltage responses and control signals using the SISO and MISO controllers: (a) 2 A load, (b) $3 \mathrm{~A}$ load, (c) $4 \mathrm{~A}$ load and (d) $2 \mathrm{~A} \rightarrow 3 \mathrm{~A} \rightarrow 4 \mathrm{~A}$ load. 
(a) load current set 3 A, output voltage set $7 v \rightarrow 8 v \rightarrow 9 v \rightarrow 8 v \rightarrow 7 v$
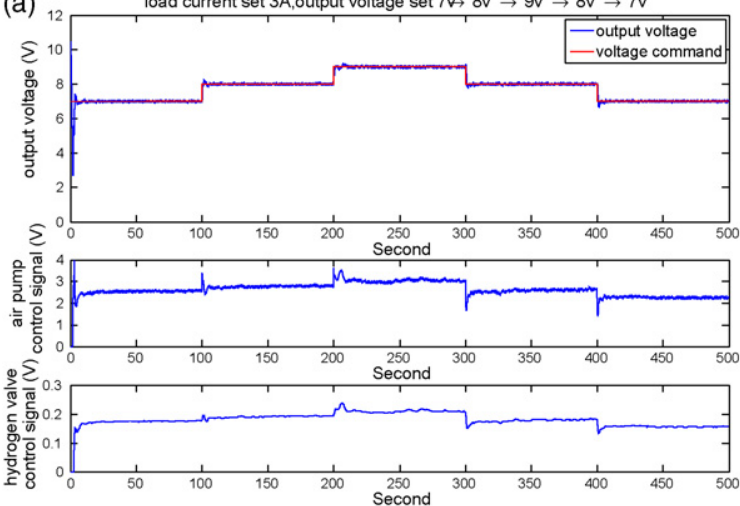

(c)
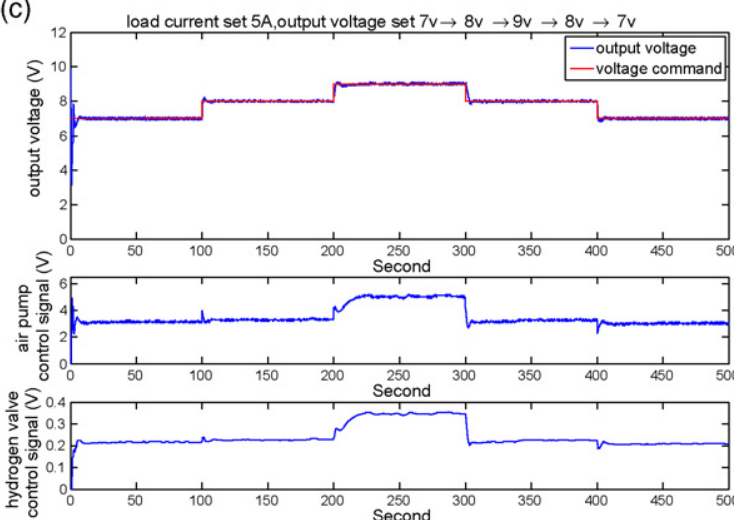
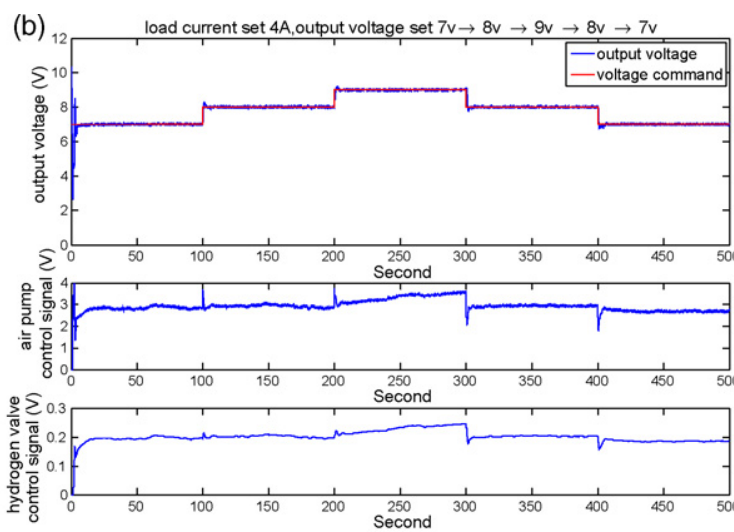

(d) load current set 6 A, output voltage set $7 v \rightarrow 8 v \rightarrow 9 v \rightarrow 8 v \rightarrow 7 v$
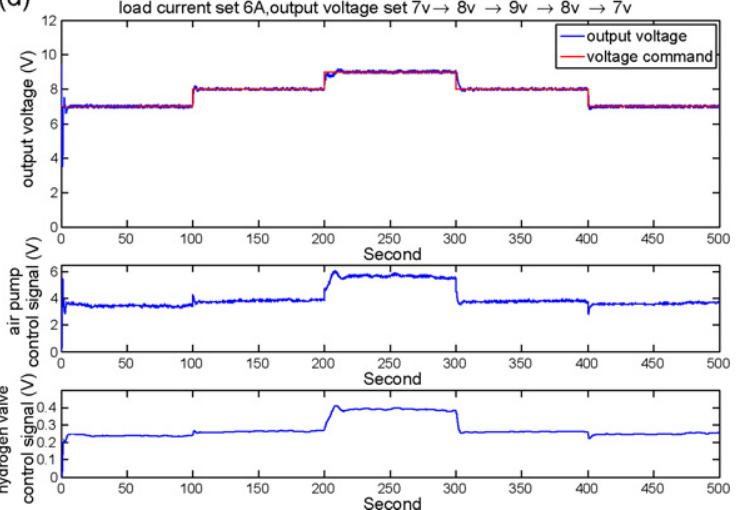

Fig. 14. The voltage responses and control signals, with settings of $7 \mathrm{~V} \rightarrow 8 \mathrm{~V} \rightarrow 9 \mathrm{~V} \rightarrow 8 \mathrm{~V} \rightarrow 7 \mathrm{~V}$ : (a) $3 \mathrm{~A}$ load, (b) $4 \mathrm{~A}$ load, (c) $5 \mathrm{~A}$ load and (d) $6 \mathrm{~A}$ load.

note that for the SISO system [22], the hydrogen consumption was set as 1.2 liter-per-minute (LPM) in order to achieve system stability and performance. Using the proposed multivariable controller, the hydrogen consumption can be reduced to about 0.6 LPM.

To eliminate the steady-state error, $W_{1}^{\prime} K_{23}^{\prime}(z)$ was designed and implemented. The experimental results are shown in Figs. 13 and 14. In Fig. 13, we set the output voltage at $9.5 \mathrm{~V}$, with the fixed current settings $2 \mathrm{~A}, 3 \mathrm{~A}$ and $4 \mathrm{~A}$ in (a), (b) and (c), respectively, and a varied current loading of $2 \mathrm{~A} \rightarrow 3 \mathrm{~A} \rightarrow 4 \mathrm{~A}$ in (d). The corresponding output voltage and hydrogen consumption are compared with the SISO study in Ref. [22]. First of all, the MISO and SISO robust controllers achieved similar voltage responses. On the other hand, the hydrogen consumption was significantly reduced by the multivariable robust controller. For quantitative comparison, Table 3 illustrates the RMS error of the output voltage and the average duty ratio of the hydrogen valve calculated from Fig. 13. It is noted that the hydrogen consumption was reduced to about 20-30\%, as compared to the SISO controller (see Appendix A for experimental verification). That is, the hydrogen consumption was regulated according to the current loads to avoid waste of fuel. To conclude, the designed controller has not only achieved robust performance for the closed-loop system, but also exhibits reduced hydrogen consumption.

In Fig. 14, we set the voltage command as $7 \mathrm{~V} \rightarrow 8 \mathrm{~V} \rightarrow 9 \mathrm{~V} \rightarrow 8 \mathrm{~V} \rightarrow 7 \mathrm{~V}$, with current loads of $3 \mathrm{~A}$, $4 \mathrm{~A}, 5 \mathrm{~A}$ and $6 \mathrm{~A}$, respectively. Firstly, the controller demon-

Table 3

Statistic data from Fig. 13

\begin{tabular}{|c|c|c|c|c|c|c|}
\hline & \multirow{2}{*}{$\frac{2 \mathrm{~A} \mathrm{(a)}}{20 \mathrm{~s} \rightarrow 300 \mathrm{~s}}$} & \multirow{2}{*}{$\begin{array}{l}3 \mathrm{~A}(\mathrm{~b}) \\
20 \mathrm{~s} \rightarrow 300 \mathrm{~s}\end{array}$} & \multirow{2}{*}{$\frac{4 \mathrm{~A}(\mathrm{c})}{20 \mathrm{~s} \rightarrow 300 \mathrm{~s}}$} & \multicolumn{3}{|c|}{$2 \mathrm{~A} \rightarrow 3 \mathrm{~A} \rightarrow 4 \mathrm{~A}(\mathrm{~d})$} \\
\hline & & & & $20 \mathrm{~s} \rightarrow 100 \mathrm{~s}$ & $100 \mathrm{~s} \rightarrow 200 \mathrm{~s}$ & $200 s \rightarrow 300 s$ \\
\hline \multicolumn{7}{|l|}{ MISO } \\
\hline RMS error & 0.0504 & 0.0366 & 0.0365 & 0.0142 & 0.0361 & 0.0371 \\
\hline Average air pump voltage (V) & 2.5953 & 3.1921 & 3.7702 & 2.3692 & 3.1905 & 3.7959 \\
\hline Average hydrogen flow rate (LPM) & 0.24 & 0.294 & 0.36 & 0.237 & 0.3046 & 0.3556 \\
\hline \multicolumn{7}{|l|}{ SISO } \\
\hline RMS error & 0.023 & 0.038 & 0.033 & 0.029 & 0.063 & 0.049 \\
\hline Average air pump voltage (V) & 2.06 & 3.425 & 3.2 & 2.33 & 2.74 & 3.78 \\
\hline Average hydrogen flow rate (LPM) & 1.2 & 1.2 & 1.2 & 1.2 & 1.2 & 1.2 \\
\hline
\end{tabular}


Table 4

Statistic data from Fig. 14 (with settings of $7 \mathrm{~V} \rightarrow 8 \mathrm{~V} \rightarrow 9 \mathrm{~V} \rightarrow 8 \mathrm{~V} \rightarrow 7 \mathrm{~V}$ )

\begin{tabular}{|c|c|c|c|c|c|}
\hline & $7 \mathrm{~V}$ & $8 \mathrm{~V}$ & $9 \mathrm{~V}$ & $8 \mathrm{~V}$ & $7 \mathrm{~V}$ \\
\hline & $20 \mathrm{~s} \rightarrow 100 \mathrm{~s}$ & $100 \mathrm{~s} \rightarrow 200 \mathrm{~s}$ & $200 \mathrm{~s} \rightarrow 300 \mathrm{~s}$ & $300 \mathrm{~s} \rightarrow 400 \mathrm{~s}$ & $400 \mathrm{~s} \rightarrow 500 \mathrm{~s}$ \\
\hline \multicolumn{6}{|c|}{ RMS error } \\
\hline $4 \mathrm{~A}$ & 0.0177 & 0.0506 & 0.0494 & 0.0924 & 0.0772 \\
\hline $5 \mathrm{~A}$ & 0.0146 & 0.0489 & 0.0658 & 0.116 & 0.0683 \\
\hline $6 \mathrm{~A}$ & 0.0227 & 0.0507 & 0.0772 & 0.1024 & 0.0641 \\
\hline $4 \mathrm{~A}$ & 2.8444 & 2.9217 & 3.3226 & 2.9188 & 2.6826 \\
\hline $5 \mathrm{~A}$ & 3.1441 & 3.2974 & 4.9313 & 3.222 & 3.0157 \\
\hline $6 \mathrm{~A}$ & 3.4375 & 3.8177 & 5.6203 & 3.7858 & 3.6014 \\
\hline \multicolumn{6}{|c|}{ Average hydrogen flow rate (LPM) } \\
\hline $3 \mathrm{~A}$ & 0.237 & 0.2376 & 0.2424 & 0.2376 & 0.2376 \\
\hline
\end{tabular}
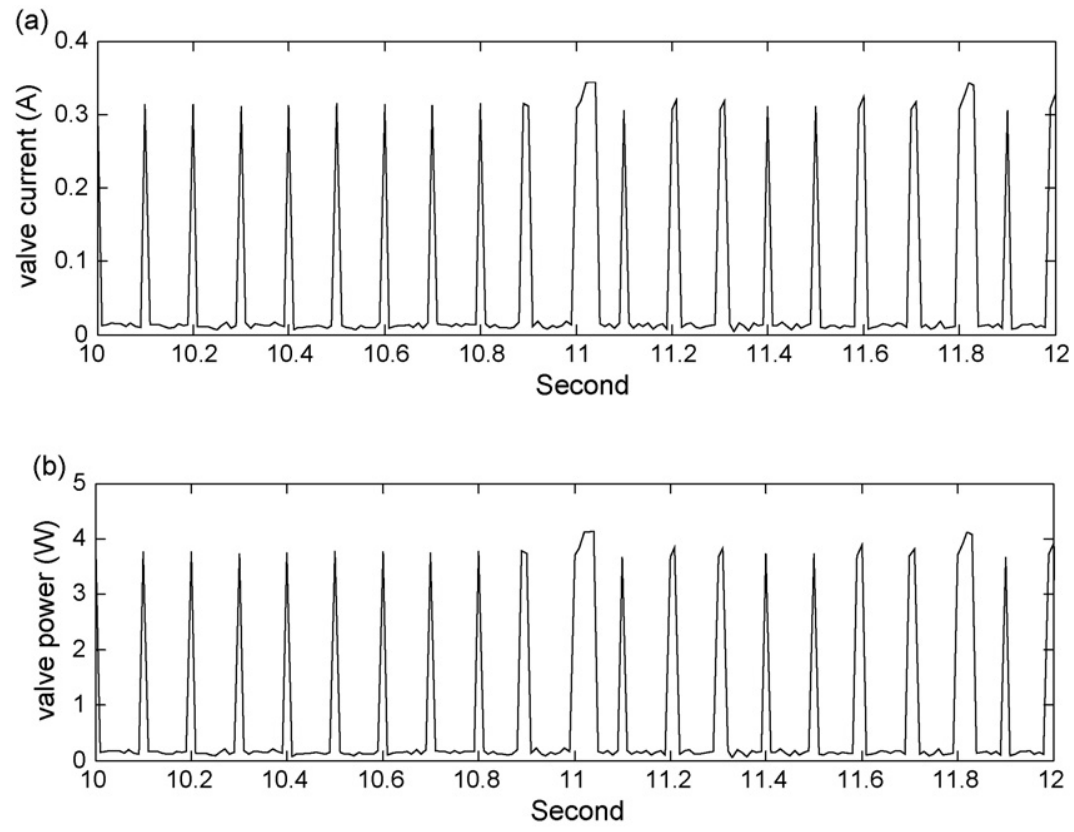

Fig. 15. Dissipated power of the hydrogen valve. (a) Valve current measurement and (b) valve power measurement.

strated excellent tracking ability with the voltage command. Furthermore, the statistic data from Fig. 14 is shown in Table 4, where the hydrogen consumption was significantly reduced from 1.2 LPM to about 0.24-0.47 LPM. And more hydrogen was supplied to the system when the power load was increased.

\section{Conclusion}

In this paper, multivariable robust controllers have been designed and implemented on a PEMFC system. At first, the dynamics of the PEMFC was described and modeled as a MIMO system. By fixing the output resistance, we have succeeded in controlling the output voltage by regu-
Table 5

Integrated flow with various duty ratios

\begin{tabular}{lc}
\hline Duty ratio $(\%)$ & Liter \\
\hline 20 & 8 \\
30 & 11 \\
40 & 14 \\
50 & 18 \\
60 & 23 \\
70 & 27 \\
80 & 32 \\
90 & 36 \\
\hline
\end{tabular}


Table 6

Integrated flow rate with MISO system

\begin{tabular}{lllll}
\hline Loadings & SISO & & \multicolumn{2}{l}{ MISO } \\
\cline { 2 - 2 } \cline { 5 - 5 } & $\begin{array}{l}\text { Expect Flow } \\
\text { (Liter) }\end{array}$ & & $\begin{array}{l}\text { Expect Flow } \\
\text { (Liter) }\end{array}$ & $\begin{array}{l}\text { Measured flow } \\
\text { (Liter) }\end{array}$ \\
\hline $9.5 \mathrm{~V}, 2 \mathrm{~A}$ & 37.33 & & 7.47 & 7 \\
$9.5 \mathrm{~V}, 3 \mathrm{~A}$ & 37.33 & 9.15 & 9 \\
$9.5 \mathrm{~V}, 4 \mathrm{~A}$ & 37.33 & 11.2 & 11 \\
\hline
\end{tabular}

lating the hydrogen and airflow rates through the designed controllers. The experimental results showed that using a suitable weighting function, the proposed multivariable robust controller not only achieved robust performance, but also significantly reduced the hydrogen consumption of the PEMFC system.

\section{Acknowledgments}

The authors would like to thank Delta Electronics ${ }^{\mathrm{TM}}$ and CSIST for providing the portable PEMFC system studied in this paper.

\section{Appendix A}

A couple of experiments were designed to verify the reduction of hydrogen consumption and energy dissipation. For the first experiment, we aimed to show that hydrogen consumption could be evaluated by the duty ratio of the PWM signal. Setting the inlet flow rate to 8 LPM with duration of $5 \mathrm{~min}$, we applied control signals with various duty ratios to the hydrogen valve and measured the flow rates with a digital flow meter SMC-PF2A710 [32]. The results in Table 5 illustrated that the hydrogen consumption can be evaluated by the duty ratio of the PWM signal. Furthermore, we applied the measured hydrogen control signals in Fig. 13 to control the hydrogen valve. Given the inlet flow of 8 LPM, the expected flow was calculated and compared with the measured flow, as shown in Table 6. The results showed that the consumed gas flow was as expected, with little difference because the flow meter can only give integer readings.

For the second experiment, we verified that the energy dissipation of the hydrogen valve is reduced by the proposed multivariable robust controller. Using a current sensor (Hall sensor), we measured the operating current and power consumption of the hydrogen valve as shown in Fig. 15. It is illustrated that the energy dissipation of the MISO system is less than the SISO system (where the valve was fully open and hydrogen was supplied in a constant rate). From the above experiments, the proposed multivariable robust controller can really reduce both hydrogen consumption and energy dissipation.

\section{References}

[1] T.P. Jay, H. Peng, A.G. Stefanopoulou, J. Dyn. Syst. Meas. Control 126 (2004) 14-25.

[2] D. Chen, H. Peng, J. Dyn. Syst. Meas. Control 127 (2005) 424-432.

[3] A. Forrai, H. Funato, Y. Yanagita, Y. Kato, IEEE Trans. Energy Convers. 20 (2005) 668-675.

[4] A. Kazim, P. Lund, Proceedings of the Institution of Mechanical Engineers, 2006, pp. 847-853.

[5] C. Wang, M.H. Nehrir, H. Gao, IEEE Trans. Energy Convers. 21 (2006) 586-595.

[6] C.H. Woo, J.B. Benziger, Chem. Eng. Sci. 62 (2007) 957-968.

[7] A.P. Vega-Leal, F.R. Palomo, F. Barragan, C. Garcia, J.J. Brey, J. Power Sources 169 (2007) 194-197.

[8] R.N. Methekar, V. Prasad, R.D. Gudi, J. Power Sources 165 (2007) 152-170.

[9] P. Rodatz, G. Paganelli, L. Guzzella, Proceedings of the American Control Conference, 2003, pp. 2043-2048.

[10] A. Di Domenico, A. Miotti, M. Alhetairshi, Y.G. Guezennec, S.S.V. Rajagopalan, S. Yurkovich, Proceedings of the 2006 American Control Conference, 2006, pp. 478-483.

[11] K. Sedghisigarchi, A. Feliachi, Proceedings of the 35th Southeastern Symposium on System Theory, 2003, pp. 464-467.

[12] P. Thounthong, S. Rael, B. Davat, J. Power Sources 158 (2006) 806814.

[13] H.S. Lee, K.S. Jeong, B.S. Oh, Int. J. Hydrogen Energy 28 (2003) 215222.

[14] F. Jurado, J.R. Saenz, IEEE Trans. Energy Convers. 18 (2003) 342347.

[15] R.J. Wai, L.W. Liu, R.Y. Duan, IEEE Trans. Ind. Electron. 53 (2006) 272-280.

[16] F. Zenith, S. Skogestad, J. Process Control 17 (2007) 333-347.

[17] Z. Jiang, L. Gao, M.J. Blackwelder, R.A. Dougal, J. Power Sources 130 (2004) $163-171$.

[18] T.T. Georgiou, M.C. Smith, IEEE Trans. Autom. Control 35 (1990) 673-686.

[19] G. Vinnicombe, IEEE Trans. Autom. Control 38 (1993) 1371-1383.

[20] K. Zhou, J.C. Doyle, K. Glover, Robust Optim. Control (1996) 596.

[21] F.C. Wang, Y.P. Yang, H.P. Chang, Y.W. Ma, C.W. Huang, B.J. Weng, Proceedings of 4th International ASME Conference on Fuel Cell Science, Engineering and Technology, 2006, p. 6.

[22] F.C. Wang, Y.P. Yang, C.W. Huang, H.P. Chang, H.T. Chen, J. Power Sources 164 (2007) 704-712.

[23] Y.P. Yang, F.C. Wang, H.P. Chang, Y.W. Ma, B.J. Weng, J. Power Sources 164 (2007) 761-771.

[24] M. Ceraolo, C. Miulli, A. Pozio, J. Power Sources 113 (2003) 131-144.

[25] J.M. Maciejowski, B.C. Chang, Appl. Mech. Rev. 44 (1991) 114.

[26] I. Goethals, K. Pelckmans, J.A.K. Suykens, M. Bart De, IEEE Trans. Autom. Control 50 (2005) 1509-1519.

[27] E.E. Topcu, I. Yuksel, Z. Kamis, Mechatronics 16 (2006) 365-378.

[28] MAC-VALVES: http://www.macvalves.com/home.html.

[29] T.T. Georgiou, M.C. Smith, IEEE Trans. Autom. Control 37 (1992) $1133-1143$.

[30] D. McFarlane, K. Glover, IEEE Trans. Autom. Control 37 (1992) 759-769.

[31] S. Skogestad, Multivariable Feedback Control: Analysis and Design, Wiley, New York, 1996, pp. 352-362.

[32] SMC-digital flow meter: http://www.smcworld.com. 